\title{
Impact of the Coronavirus Pandemic on Emergency Department (ED) Visits for Ob/Gyn Care
}

\author{
Sepideh Mehri*, Robert Berg, Iffath Abbasi Hoskins \\ Department of Obstetrics and Gynecology, New York University Langone Health, New York, USA \\ Email address: \\ Sepideh.Mehri@nyulangone.org (S. Mehri), Robert.Berg@nyulangone.org (R. Berg), Ifffath.Hoskins@nyulangone.org (I. A. Hoskins) \\ ${ }^{*}$ Corresponding author
}

To cite this article:

Sepideh Mehri, Robert Berg, Iffath Abbasi Hoskins. Impact of the Coronavirus Pandemic on Emergency Department (ED) Visits for Ob/Gyn Care. Journal of Gynecology and Obstetrics. Vol. 9, No. 2, 2021, pp. 42-45. doi: 10.11648/j.jgo.20210902.13

Received: March 10, 2021; Accepted: March 19, 2021; Published: March 26, 2021

\begin{abstract}
The COVID-19 pandemic has disrupted currently held norms and best practices for women who require evaluations and interventions for Obstetrics and gynecology $(\mathrm{Ob} / \mathrm{Gyn})$ emergency care. The resulting paradigm shifts have demonstrated a reassessment of how $\mathrm{Ob} / \mathrm{Gyn}$ emergency care is provided in an acute setting while avoiding potential short and long-term harm. The objective of this study is to retrospectively evaluate if patients self-selected to avoid Emergency Department (ED) visits for perceived minor complaints and if postponing such care increased morbidity. Patients accessing Ob/Gyn ED care during the Covid-19 "lockdown period" (group A) in New York State were compared with those during a similar time frame in 2019 (group B). Primary outcomes were the number of ED visits and admission diagnoses. Secondary outcomes were the number of surgeries and clinical acuity on presentation. There was a $72 \%$ reduction in the total number of ED visits in group A compared to group B. Although the majority of the visits were for abdominal pain and vaginal bleeding in both groups, among patients who presented with vaginal bleeding in group A, a significantly higher number was due to early pregnancy loss rather than non-pregnancy-related uterine bleeding. The number of visits for non-emergency visits such as vulvovaginitis and contraceptive management was significantly lower and absent respectively. There were $61.5 \%$ fewer cases requiring emergency surgery in group A, however, the case acuity remained high with many patients being clinically unstable at presentation. There was a nonsignificant trend of an increased number of surgeries for ectopic pregnancy and pregnancy loss in group A compared to group B. Non-emergency surgeries, and visits for pain control, wound infections or ileus were absent in group A. We conclude that the decline in the ED visits and total number of emergency surgeries during the "lockdown period "were secondary to patients' choices. This decrease was more pronounced for minor indications; however, many patients with serious conditions had delayed ED visits until they were clinically unstable. Therefore a reassessment of how patients access $\mathrm{Ob} / \mathrm{Gyn}$ care via $\mathrm{ED}$ is indicated, with the goal being to avoid visits for minor indications while preventing potential life-threatening complications.
\end{abstract}

Keywords: COVID-19, Lockdown Period, Ob/Gyn Visits, Emergency Surgery

\section{Introduction}

Emergency Departments (EDs) are a significant resource for medical care in the United States. In 2017, there were 139 million ED visits. [1] Approximately $10.4 \%$ of these visits resulted in hospital admissions. In general, socioeconomic and insurance status affect ED usage. For uninsured and publicly insured patients, the ED is the primary source of medical care and admission to the hospital. This is in contrast to those with private insurance, as they are more likely to be directly admitted to the hospital from a doctor's office or clinic. [2] ED utilization reflects the health needs of the surrounding community and often may provide the only available care for individuals who cannot or choose not to obtain care elsewhere. [3] Many ED visits are a burden on limited resources and potentially preventable. Therefore, providing access to high-quality, community-based health care could potentially prevent the need for a significant proportion of current ED visits.

On March 13, 2020, the United States declared a national emergency due to the Coronavirus pandemic. New York was one of the first states to report a confirmed case of Covid-19. 
Following this, a statewide shelter-in-place Government order was instituted on March 19, 2020.

As the number of patients hospitalized with COVID-19 increased, early reports from New York City (NYC) suggested sharp drops in the numbers of patients seeking emergency medical care for other reasons. Overall, ED volume declined by approximately $42 \%$. [5, 7, 16] The underlying reasons were 2-fold: firstly, patients were avoiding the ED because of fear of contracting coronavirus, regardless of whether an underlying medical emergency existed or not. This likely resulted in increased morbidity and mortality. [9] Secondly, the public was advised by health care professionals and by government officials to avoid hospitals and other health care facilities in an effort to decrease/avoid contact with potentially infected patients and to avoid overburdening the EDs. [5, 6, 10]

The aim of this study was twofold, 1. To determine whether patients self-selected to stay home for $\mathrm{Ob} / \mathrm{Gyn}$ complaints during the Coronavirus pandemic and 2. If postponing the ED visits resulted in increased numbers of acute cases and/or emergencies requiring surgical interventions.

\section{Methods}

After IRB approval, all $\mathrm{Ob} / \mathrm{Gyn}$ visits and admissions to the NYU Langone Medical Center ED between March 23 and May 25, 2020, were reviewed for this study. Admission diagnosis and admission type (minor vs. major and emergency vs. non-emergency) were reviewed.

The time span between March 23 and May 25, 2020, was defined as the "lockdown period". All ED visits for Ob/Gyn patients during this time were assessed and were compared with a similar time frame in 2019 (March 25 to May 27, 2019). Each patient was categorized by admitting diagnosis and was further subdivided into whether she had a minor or a major emergency (Table 1). Major emergencies were defined as those requiring immediate/urgent surgical treatment for the admitting diagnosis. (Table 2).

Table 1. Diagnostic criteria.

\begin{tabular}{|c|c|c|c|}
\hline Diagnostic Criteria & Group A n=218 & Group B n=771 & p-Value \\
\hline Abdominal Pain & $137(62 \%)$ & $440(57 \%)$ & 0.1472 \\
\hline Vaginal Bleeding & $48(22 \%)$ & $153(19.8 \%)$ & 0.5425 \\
\hline Pregnancy-related issues & $34(71 \%)$ & $89(58 \%)$ & $<0.001$ \\
\hline (missed/threatened $\mathrm{AB}$, retained $\mathrm{POC}$ ) & $9(19 \%)$ & $59(38 \%)$ & $<0.001$ \\
\hline AUB & $5(10 \%)$ & $5(3 \%)$ & 1 \\
\hline Bartholin/Vulvar Abscess & $2(0.9 \%)$ & $6(0.8 \%)$ & 1 \\
\hline Vaginal Discharge, Vulvovaginitis & $2(0.9 \%)$ & $30(4 \%)$ & 0.048 \\
\hline Ectopic Pregnancy/PUL (Major) & $10(4.5)$ & $19(2.5 \%)$ & 0.1577 \\
\hline Ovarian Cyst Rupture/torsion (Major) & $6(2.7 \%)$ & $8(1 \%)$ & 0.117 \\
\hline IUD Complication & 0 & $3(0.4 \%)$ & 0.822 \\
\hline OHSS & 0 & $2(0.25 \%)$ & 1 \\
\hline Pelvic Infection/Pelvic Inflammatory Disease & $1(0.5 \%)$ & $13(1.6 \%)$ & 0.3039 \\
\hline Other (Trauma, Contraception, Post-operative Complication) & $10(4.5 \%)$ & $89(11.5 \%)$ & 0.0030 \\
\hline
\end{tabular}

AUB: abnormal uterine bleeding, PUL: Pregnancy of unknown location, TOA: tubo- ovarian abscess, OHSS: ovarian hyper-stimulation syndrome, AB abortion, POC: Products of conception.

Table 2. Total surgeries for major emergencies.

\begin{tabular}{llll}
\hline Total Surgeries & Group A (n=10) & Group B (N=26) & p-Value \\
\hline Ovarian Cyst Rupture/Torsion & $4(40 \%)$ & $13(50) \%$ & 0.7169 \\
Ectopic pregnancy & $3(30 \%)$ & $5(19.2 \%)$ & 0.6576 \\
Missed/Incomplete Abortion & $3(30 \%)$ & $5(19.2 \%)$ & 0.6576 \\
AUB & 0 & $2(7.6 \%)$ & 1 \\
Vulvar Abscess & 0 & $1(3.8 \%)$ & 1 \\
Clinically Unstable & $7(70 \%)$ & $8(31 \%)$ & 0.0432 \\
\hline
\end{tabular}

Clinically unstable: patients requiring blood transfusion and/or immediate surgery; AUB: abnormal uterine bleeding

\section{Statistical Analysis}

All clinical parameters were analyzed retrospectively. Data analysis proceeded anonymously and is expressed as mean \pm standard deviation (SD). Chi-square and Fisher's exact tests were used, and a value of $\mathrm{p}<0.05$ was statistically significant.

\section{Results}

During the "lockdown period" there were 218 Ob/Gyn patient visits to the ED (group A), versus 771 visits in a similar time period in 2019 (group B). There was a 72\% reduction in ED visits in group A when compared to group B. The most common reason for the ED visit in groups $A$ and $\mathrm{B}$, was abdominal pain due to gynecologic reasons, (62\% and $57 \%$ respectively, $\mathrm{p}=0.147)$. The second most common reason for the ED visit in both groups was vaginal bleeding, ( $22 \%$ vs $19.8 \%$ respectively, $p=0.542$ ). Of those who presented with vaginal bleeding, significantly more women in group A had pregnancy- 
related issues such as incomplete abortion or retained products of conception $(71 \%$ vs $58 \%, \mathrm{p}<0.001)$ and significantly fewer patients had AUB secondary to abnormal menses or fibroids, when compared to those in group B $(19 \%$ vs $38 \%, \mathrm{p}<0.001)$. There were more patients in group A than in group B with complaints related to ovarian torsion and ectopic pregnancy $(2.7 \%$ vs $1 \%$ and $4.5 \%$ vs $2.5 \%$ respectively), although this was not statistically significant. Fewer women in group A had ED visits for vulvovaginitis and contraception issues when compared to those in group B. This was statistically significant ( $p=0.048$ and 0.0039 respectively). There were no differences in the numbers of ED visits for labial abscess/Bartholin's cyst in both groups. Overall, the number of emergency cases requiring surgery decreased by $61.5 \%$ between the 2 groups. (Table 2). The most common indication for surgery in both groups was ovarian pathology. Fewer women in group A required diagnostic laparoscopy for abdominal pain $(40 \%$ vs $50 \%, \mathrm{P}=0.7169)$. A higher percentage required surgery for ruptured ectopic pregnancy in group A versus in group B. This was not statistically significant ( $30 \%$ vs $19 \%, \mathrm{P}=0.657$ ). A greater proportion of women in Group A required dilatation and curettage (D\&C) for heavy bleeding due to early pregnancy loss, versus in group B. This was not statistically significant $(30 \%$ vs $19 \%, \mathrm{P}=0.0657)$. Of the women in group A undergoing surgery for adnexal pathology or ectopic pregnancy, there was a significantly higher percentage who presented as clinically unstable, exhibiting abnormal vital signs and hem peritoneum and they required longer hospitalizations versus those in group B $(70 \%$ vs $31 \%, p<0.0432)$. There were no admissions in group A for contraceptive issues or pelvic trauma nor were there ED visits for any postoperative complications.

\section{Discussion}

To our knowledge, this is the first documentation of the impact of the Coronavirus pandemic and the resulting Government regulations on $\mathrm{Ob} / \mathrm{Gyn}$ visits to and related hospital admissions from the ED. Our analysis indicates that the overall number of ED visits for $\mathrm{Ob} / \mathrm{Gyn}$ care dramatically declined for both minor and major indications during the "lockdown period" of the Coronavirus pandemic.

The decrease in ED volume was more pronounced for minor indications such as contraceptive needs, vulvovaginitis, and urinary tract infections. We hypothesize that patients likely chose to avoid seeking ED care for conditions that they felt could be self-managed, and/or did not cause severe distress to them.

Interestingly, despite the significantly lower volume of ED visits, the acuity of the $\mathrm{Ob} / \mathrm{Gyn}$ cases during the "lockdown period" remained unchanged and even increased in some instances, such as in patients with ovarian torsion or ectopic pregnancy. They had a delayed presentation to the ED, after their condition had worsened.

In our analysis, there was a non-significant increase in the number of patients requiring emergency surgery for life- threatening indications such as ruptured ectopic pregnancy during the "lockdown period" (group A). Additionally, a majority of the patients undergoing emergency Ob/Gyn surgery in group A were clinically unstable $(p=0.043)$. One possibility is that patients may have self-selected to avoid seeking $\mathrm{Ob} / \mathrm{Gyn}$ care in the ED if they considered their condition to be minor, and the patients who ultimately needed emergency gynecological surgery likely sought care after their condition had progressed or worsened. Our sub-analysis revealed that, of the women who required surgery, all were emergencies that could not have been managed conservatively.

In this study, the most common indication for ED visits was abdominal pain due to gynecologic pathology. Our findings are similar to those of Weiss et al who reviewed the characteristics of ED visits in the US, and found that females had a higher overall rate of ED visits vs. males and that abdominal pain was among the top 5 causes of such visits. [4] Our observations are also consistent with the reported declines in inpatient admissions in other specialties such as Medicine and Neurology during the Coronavirus pandemic. [12-14]. Investigators in Italy and Germany reported that patients with minor strokes and transient ischemic attacks arrived late, and in a more acute condition to the ED, thus resulting in increased complication rates. [8, 11, 15].

ED visits may have been avoided by patients with gynecologic complaints for various reasons, including fear of contracting the virus in the hospital setting and following the guidelines established by Government leaders to avoid burdening the health care system. As such, women may have chosen to tolerate the discomfort of non-acute conditions such as vulvovaginitis or pelvic infections while following the guidelines for social distancing and sheltering in place. Limitations of the current study include its retrospective, observational design, and the analysis being from a single, urban medical center. The strengths of the study are that it is the first of its kind to describe how women prioritized and managed their own Ob/Gyn visits to the ED during the "lockdown period" of the Coronavirus pandemic.

\section{Conclusions}

Based on our findings, many visits for gynecologic complaints may not require care in an ED during nonpandemic periods, but rather may be managed using telehealth modalities in Urgent Care or Walk-In clinic settings. This option would allow for the scarce and expensive ED resources to be allocated to more acute and serious health-related $\mathrm{Ob} / \mathrm{Gyn}$ conditions.

We suggest that the information in this study could be utilized to develop a contingency plan during national emergencies so that women with serious, acute conditions can have access to the scarce ED resources in a timely fashion while avoiding potentially life-threatening complications.

\section{Acknowledgements}

Pertab Dorothy, Anna Davis, Steven Friedman. 


\section{References}

[1] Rui P., Kang K., National Hospital Ambulatory Medical Care Survey: 2017 emergency department summary tables. National Center for Health Statistics. https://www.cdc.gov/nchs/data/nhamcs/web_tables/2017_ed_ web_tables-508.pdf. http://hcupnet.ahrq.gov/HCUPnet.jsp.

[2] Morganti-Gonzalez K, Baufman S, Blanchard J, Abir M, Iyer $\mathrm{N}$, Smith A, et al. The Evolving Role of Emergency Departments in the United States. RAND RR 280-ACEP. Santa Monica, CA: Rand Corp; May, 2013. www.rand.org.

[3] Ning Tang, MD, John Stein, MD, Renee Y. Hsia, MD, MSc, Judith H. Maselli, MSPH, and Ralph Gonzales, MD, MSPH. Trends and Characteristics of US Emergency Department Visits, 1997-2007. JAMA. 2010 Aug 11; 304 (6): 664-670. doi: 10.1001/jama.2010.1112.

[4] Audrey J Weiss, PhD, Lauren M Wier, MPH, Carol Stocks, $\mathrm{PhD}, \mathrm{RN}$, and Janice.

[5] Blanchard, MD, PhD. An Overview of Emergency Department Visits in the United States, 2011.: Healthcare Cost and Utilization Project (HCUP) Statistical Briefs. June 2014. Find NCBI SARS-CoV-2 literature, sequence, and clinical content: https://www.ncbi.nlm.nih.gov/sars-cov-2/.

[6] Wong LE, Hawkins JE, Langness S, Murrell KL, Iris P, Sammann A. Where are all the patients? Addressing Covid-19 fear to encourage sick patients to seek emergency care. NEJM Catalyst Innovations in Care Delivery 2020. Epub May 14, 2020. https://catalyst.nejm.org/doi/full/10.1056/CAT.20.0193.

[7] Kathleen P. Hartnett, PhD; Aaron Kite-Powell, MS; Jourdan DeVies, MS; Michael A. Coletta, MPH; Tegan K. Boehmer, $\mathrm{PhD}$, Jennifer Adjemian, $\mathrm{PhD}$, Adi V. Gundlapalli, $\mathrm{MD}, \mathrm{PhD}$; National Syndromic Surveillance Program Community of Practice. CDC. Impact of the COVID-19 Pandemic on Emergency Department Visits - United States, January 1, 2019-May 30, 2020.

[8] US national Library of Medicine. Centrai Trial. g ov. The Impact of the Covid-19 Outbreak on Emergency Room Attendances of Surgical Patients.

[9] Jorge H. Nuñez, Andrea Sallent, Kushal Lakhani, Ernesto Guerra-Farfan, Nuria Vidal, Seper Ekhtiari, and Joan Minguel. Impact of the COVID-19 Pandemic on an Emergency Traumatology Service: Experience at a Tertiary Trauma Centre in Spain. NCBI, 2020 Jul; 51 (7): 1414-1418.

[10] Wang D, Hu B, Hu C, Zhu F, Liu X, Zhang J. Clinical Characteristics of 138 Hospitalized Patients with 2019 Novel Coronavirus-Infected Pneumonia in Wuhan, China. JAMA. 2020 Feb 7.

[11] World Health Organization (WHO). Novel Coronavirus (2019- nCoV) situation reports. 2020. https://www.who.int/emergencies/diseases/novel-coronavirus2019/situation-reports (02 April 2020).

[12] Mohammad Hassan A. Noureldine, Elliot Pressman, Paul R. Krafft, Mark S. Greenberg, Siviero Agazzi, Harry van Loveren, Puya Alikhani. Impact of the COVID-19 Pandemic on Neurosurgical Practice at an Academic Tertiary Referral Center: A Comparative Study. 2020 Jul; 139: e872-e876.

[13] Molly M. Jeffery, PhD; Gail D’Onofrio, MD, MS; Hyung Paek, MD; et al. Trends in Emergency Department Visits and Hospital Admissions in Health Care Systems in 5 States in the First Months of the COVID-19 Pandemic in the US. JAMA Intern Med. 2020; 180 (10): 1328-1333.

[14] Rosenbaum L. The untold toll: the pandemic's effects on patients without COVID-19. N Engl J Med. 2020; 382 (24): 2368-2371.

[15] De Filippo O, D’Ascenzo F, Angelini F, et al. Reduced rate of hospital admissions for ACS during COVID-19 outbreak in Northern Italy. N Engl J Med. 2020; 383: 88-89.

[16] Jeffery MM, D’Onofrio GD, Paek Y, et al. Trends in ED Visits and Hospital Admissions During COVID-19 Pandemic. American College of cardiology. Aug 05, 2020. 